\title{
¿QuÉ CITAN LOS CITADOS?: NICHOS DE CONOCIMIENTO EN EL SISTEMA \\ INTERAMERICANO DE PROTECCIÓN DE DERECHOS HUMANOS
}

What do the quoters quote?: Niches of knowledge in the Inter-American System for the protection of human rights

\author{
Efrén Guerrero Salgado \\ Camila Cedeño \\ Pontificia Universidad Católica del Ecuador
}

\section{Resumen}

El presente documento busca, a través de un estudio cualitativo-cuantitativo en torno al análisis de redes, examinar los nichos epistémicos existentes en la Corte Interamericana de Derechos Humanos, centrándose en el último periodo de sesiones, que de acuerdo a las teorías incrementales contaría con la jurisprudencia más avanzada. Se busca comprobar mediante la metodología de análisis de redes sociales, si la elección de las sentencias para dar soporte al ratio decidendi de la Corte Interamericana obedece a una elección racional o si es una decisión temática. El texto concluye explicando que, aunque hay elementos que demuestran una elección racional, no existe en la norma algo parecido a un hilo conductor de la jurisprudencia, fuera de la expansión tradicional de derechos; y que las conexiones posibles entre casos obedecen a elementos de racionalidad limitada.

\section{Palabras clave}

Jurisprudencia / Análisis de Redes / Sistema Interamericano de Derechos Humanos / Teoría de la Elección Racional.

\section{Summary}

The present document seeks to examine, through a qualitative-quantitative study based on the analysis of social networks, the epistemic niches in the Inter-American Court of Human Rights, focusing on the last session period, which, according to incremental theories, has the most advanced jurisprudence. It seeks to verify, with the use of social network analysis, if the choice of sentences to support the decisions of the Inter-American Court obeys a rational choice or if it is a thematic decision. The text concludes by explaining that, although there are elements that show a rational decision, there does not appear to be a main theme to jurisprudence, beyond the traditional expansion of rights; and that the possible connections between the cases obey elements of limited rationality.

\section{Keywords}

Jurisprudence / Network Analysis / Inter-American Human Rights System / Theory of Rational Election.

\section{Introducción}

La tradición legal latinoamericana ha tenido a la jurisprudencia como fuente material del Derecho excepcional. De hecho, la visión sobre el valor de las decisiones de los jueces ha estado basada en la reputación personal del decisor o en la importancia del caso, más allá de la existencia de reglas de derecho obligatorias y básicas de la gestión de esa fuente como parte del sistema normativo. En el caso de la norma de fuente internacional y en especial aquellas 
relacionadas a derechos humanos, hay una creciente corriente doctrinaria, en los sistemas de raigambre occidental europeo, entre ellos el ecuatoriano, que busca construir a partir de los principios de corpus iure y efecto útil una obligatoriedad de la jurisprudencia dentro del sistema jurídico interno.

En el caso de la República del Ecuador existe una creciente tendencia a la aplicación de elementos jurisprudenciales del Sistema Interamericano de Derechos Humanos en su ordenamiento jurídico. Se puede comprobar que los operadores judiciales, la academia y los abogados y abogadas utilizan los precedentes jurisprudenciales como elemento avalador de su trabajo, con el fin de dar sostenibilidad a un razonamiento jurídico desde la legitimidad internacional. En ese escenario, existe una marginalidad en la doctrina para explicar cuáles son las bases para la elección de casos dentro de la jurisprudencia del Sistema Interamericano de protección y por qué se eligen para la toma de decisiones, unas sentencias respecto a otras. De hecho, hay una práctica de aceptación de los argumentos del SIDH como válidos per se, lo que muestra "que la jurisprudencia no se ha liberado todavía de la creencia antigua o, mejor, prehistórica en la magia verbal" (Nino, 2003, p. 12). Esa singularidad debe llevar a practicantes del Derecho a la necesidad de volver a las bases de la teoría jurídica, con el fin de explicar este fenómeno, y entender cuál es la justificación del sistema de toma de decisiones del juez internacional, tomando como caso de estudio el último periodo de sesiones de la Corte Interamericana de Derechos Humanos.

Para poder dar luces a este fenómeno el presente documento busca aportar, a través del análisis de redes de los nichos epistémicos existentes en la Corte Interamericana de Derechos Humanos, centrándose en el último periodo de sesiones. Se busca comprobar si la elección de las sentencias para dar soporte a la ratio decidendi de la Corte Interamericana obedece a una elección racional o si es una decisión temática. Para esto, el texto se dividirá en cinco secciones. La primera y la segunda harán un análisis en abstracto del valor de la jurisprudencia en el sistema latinoamericano y ecuatoriano para mostrar el estado de la situación del fenómeno, especialmente relacionado al debate respecto al valor legal y el efecto vinculante de la jurisprudencia internacional en especial la del Sistema Interamericano de Protección de Derechos Humanos (en adelante SIDH). Las restantes secciones tienen como objetivo explicar el concepto de nicho de conocimiento, el cual busca ser una unidad analítica para explicar qué es aquello que pretenden encontrar los jueces en la jurisprudencia.

\section{La jurisprudencia como fuente de Derecho: acercamiento al fenómeno}

En los primeros años de jurisprudencia el estudiante siempre se enfrenta a un modo de concebir, analizar y definir el Derecho, en base a la manera que se percibe la norma dentro de una Escuela de Derecho correspondiente, hecho que no sólo responde a una razón técnica, sino política (Kennedy, 2012, p. 27). Pero a pesar de eso, existen ciertas reglas comunes en la enseńanza del Derecho, especialmente a nivel latinoamericano. En las mallas de las facultades de Derecho de los países de tradicción occidental existen materias de Introducción al Derecho, de Teoría de la norma o equivalente, cuyo objetivo es enseńar a los estudiantes los rudimentos del sistema normativo. En ellas se manifiesta una tradición kelseniana en forma, pero con un fondo adaptado a los valores de cada contexto jurídico (López Medina, 2004) con cierta uniformidad en torno a las fuentes del Derecho, lo cual coloca a la jurisprudencia como un medio auxiliar, con ciertas excepciones de obligatoriedad, que en posiciones más modernas, al menos en el caso ecuatoriano, genera no pocas dificultades ${ }^{1}$.

\footnotetext{
${ }^{1}$ Los proveedores de información legal son categóricos al respecto, en referencia a la existencia de jurisprudencia obligatoria en ciertos casos constitucionales y el triple fallo reiterado de la Corte Nacional de Justicia (Hernández Walker, 2017): Esta 
En ese orden de cosas, los textos básicos de la formación académica plantean que "de acuerdo con la opinión más generalizada, las fuentes formales del derecho son la legislación, la costumbre y la jurisprudencia” (García Maynez, 1974, p. 50). La palabra "jurisprudencia” posee, siguiendo la teoría de García Maynez, arriba citado, dos acepciones distintas: a) conjunto de principios y doctrinas contenidas en las decisiones de los tribunales, o b) en general, la ciencia del Derecho Positivo (Clemente de Diego, 2016, p. 45). Eso sí, el valor de la misma siempre ha sido un tema de discusión, como consecuencia de un hecho humano que es un tema crítico para la gestión de lo jurídico: el margen de error existente en el operador al momento de la interpretación de la ley en relación a los distintos comportamientos humanos. $\mathrm{Al}$ respecto es clásica la frase de Robespierre: "El término jurisprudencia debe borrarse de nuestra lengua. En un Estado que tiene una Constitución, una legislación, la jurisprudencia de los Tribunales no es otra cosa que la ley" (cit. en De Otto, 1987, p. 293). Esa visión de un positivismo avanzado es aún una de las ideas básicas de la gestión de la ley. No es raro en las facultades de Derecho encontrar la idea del juez como la boca de la ley, limitando la aplicación del texto legal a las elementales principios de lógica y racionalidad.

En contraposición a esa postura tradicional, otra dirección teórica acepta a la jurisprudencia de una manera más interesante y técnicamente provechosa. Esta reconoce que la jurisprudencia no tiene sino un valor marginal en nuestro ordenamiento jurídico, y que existe una regla general de clara aplicación interna ${ }^{2}$, pero que la jurisprudencia tiene un valor amplificador y ecualizador de los contenidos de la ley. Así, potencia los contenidos legales expandiéndolos a través de mandatos de optimización (Alexy, 1988) que reconstruyen y organizan los contenidos legales que ya utilizan. Del mismo modo, sirve como un medio para ecualizar y volver a la norma organizada y adaptada a la realidad circundante:

Consideremos, por ejemplo, el problema fundamental del concepto de Derecho. Algunos autores dirán que es cosa distinta de lo que deciden los tribunales de Massachusetts o de Inglaterra, que es un sistema racional, una deducción de los principios de la época, de axiomas aceptados u otras fórmulas que pueden coincidir o no con las sentencias. Pero si nos situamos desde el punto de vista de nuestro delincuente, encontraremos que le importan poco los axiomas y las deducciones y quiere saber cómo se comportarán de hecho los tribunales de Massachusetts y de Inglaterra. Yo soy bastante de esa opinión. Las profecías de lo que efectivamente harán los tribunales, y no nada más pretencioso, son lo que yo entiendo por Derecho (Holmes, 1897).

Entonces, los jueces son capaces de utilizar el sistema normativo como un mecanismo de expansión y de mejoramiento del Derecho. Como producto humano la norma debe adaptarse, de forma que se acepte al sistema como un medio no sólo de regulación de la conducta humana, sino que, en una perspectiva más humanista, se logre valorar y celebrar jurídicamente la diversidad de la sociedad (Ferrajoli, 2004); y, además, logre mostrar zonas de la dignidad humana, valores comunes que deben ser preservados a todo nivel y sostenidos por entrar a la esfera de los "indecidible" (Ferrajoli, 2008). La manera en que se resuelve ese hecho en nuestra legislación se presenta a continuación.

\footnotetext{
sentencia pone en evidencia la profunda transformación por la que está atravesando nuestra institucionalidad; y verificamos la existencia de un suprapoder que actúa sobre la Función Judicial y la Función Legislativa. La Constitución del 2008 otorga a la Corte Constitucional la potestad de anular los fallos expedidos por la Función Judicial y además la interpretación y reforma de la ley. El problema que enfrenta el operador de justicia es ¿a quién obedezco?

${ }^{2}$ Art. 3 del Código Civil Ecuatoriano: "Sólo toca al legislador explicar o interpretar la ley de un modo generalmente obligatorio. Las sentencias judiciales no tienen fuerza obligatoria sino respecto de las causas en que se pronunciaren”.
} 


\section{El caso ecuatoriano: la jurisprudencia nacional e internacional como fuentes de derecho}

El caso ecuatoriano, como positivista tradicional, negaba por regla general un razonamiento de un juez que obligase a aplicar una norma más allá de los involucrados en el proceso, cosa que como veremos más adelante, tiene cambios con el actual diseño de la Corte Constitucional. Esto confirma un viejo aforismo del mundo jurídico: "el Universo del Juez es el expediente", lo que implica que las posibilidades infinitas de ese espacio de decisión sólo se ven justificadas para las partes en su decisión particular. Pero, como en todo espacio humano hay lugares para la excepción. Al respecto, la doctrina afirma que:

Los jueces dictan ciertas normas particulares que se llaman "sentencias judiciales". Para hacerlo tienen en cuenta determinadas normas que pueden preexistir, como una ley o una costumbre, pero también muchas veces toman en consideración, en los fundamentos de la sentencia, determinados criterios generales de razonabilidad, equidad, etcétera. Esos criterios, que no fueron dictados por un legislador ni se originaron en la costumbre de la población, pueden ser tomados en cuenta por otros jueces a quienes se les plantea posteriormente un caso análogo al resuelto originariamente. Cuando tales criterios son adoptados por los jueces como fundamento de sus sentencias y el apartarse de ellos es motivo de crítica, se da origen a ciertas normas jurídicas, que los jueces pueden considerar tanto o más obligatorias (como ocurre en los Estados Unidos y Gran Bretaña) que las leyes (Nino, 2003, p. 151).

En tal razón, no puede plantearse a priori cuál fuente se admite en un sistema jurídico particular. Eso depende, principalmente de los criterios de cómo se construye dicho sistema en torno a todo el ordenamiento legal en general, y también de cómo se estructuran las normas como consecuencias de esos preceptos básicos (Nino, 2003, p. 152). Si se presenta un escenario con una jurisprudencia con efectos erga omnes, ésta debería a) ser excepcional, b) tener, dado el contenido autorreferente ${ }^{3}$ del derecho, una autorización en el mismo derecho; y c) publicarse en el Registro Oficial para que así cumpla con la regla de derecho establecida en Art. 6 del Código Civil.

$\mathrm{Al}$ respecto, el caso de la República del Ecuador propone las siguientes reglas de conducta en el caso del valor de la jurisprudencia:

a) Regla General: negación de la jurisprudencia como regla general de jurisprudencia. Sólo tiene valor para las partes involucradas (Art. 3 Código Civil).

b) Excepción ${ }^{4}$ 1. Triple Fallo Reiterado de la Corte Nacional de Justicia: El Art. 184 de la Constitución ecuatoriana establece que es competencia de la Corte Nacional de Justicia, "desarrollar el sistema de precedentes jurisprudenciales fundamentado en los fallos de triple reiteración”, y plantea en el artículo siguiente (185) las siguientes condiciones

i. Deben ser sentencias emitidas por las salas especializadas de la Corte Nacional de Justicia

ii. Estas sentencias deben reiterar por tres ocasiones la misma opinión sobre un mismo punto,

iii. Estos fallos deben remitirse el fallo al pleno de la Corte a fin de que ésta delibere y decida en el plazo de hasta sesenta días sobre su conformidad.

\footnotetext{
${ }^{3}$ Un sistema jurídico es autorreferente o autopoiético a partir de su construcción como sistema: el procedimiento jurídico (proceso), acto jurídico (elemento), norma jurídica (estructura) y dogmática jurídica (identidad) (Teubner, 1989).

${ }^{4}$ Se proponen como excepciones, ya que son elementos nacidos de la profusión del uso de la norma internacional, que han alcanzado su positivización y generan espacios de actuación fuera de la norma de clausura del Código Civil.
} 
Como efecto, el mismo artículo propone que "deberá observar la jurisprudencia obligatoria establecida de manera precedente". Finalmente, se indica que para cambiar el criterio jurisprudencial obligatorio "la jueza o juez ponente se sustentará en razones jurídicas motivadas que justifiquen el cambio, y su fallo deberá ser aprobado de forma unánime por la sala”.

c) Excepción 2. Jurisprudencia Constitucional: El carácter vinculante de la jurisprudencia de la Corte Constitucional ecuatoriana nace de su vocación de interpretación, autorizado por la Carta Magna, supuesto en el Art. 429 del texto de Montecristi. Esta idea indica que el citado organismo debería —al menos en teoría — ser capaz de actualizar, indicar vías de acción y mejorar la capacidad de los operadores de justicia, para evitar el fenómeno de petrificación constitucional (Casal Hernández, 2004). De acuerdo con los datos disponible, la jurisprudencia de la Corte Constitucional de la República del Ecuador (en adelante CCE) ha emitido las siguientes reglas de utilidad:

\section{i) Definición:}

[...] cuando se presenta en la judicatura una demanda o acción por parte de cualquier persona, comunidad, pueblo, nacionalidad o colectivo, vulnerada en uno o más de sus derechos constitucionales, y si ese caso tiene como precedente una sentencia en la cual ya se tuteló el derecho reclamado, ha instalado condiciones de predictibilidad, por lo que, el ulterior caso necesariamente debe guardar armonía con el precedente jurisprudencial" (Sentencia CCE, 2016a; Sentencia CCE 2016b) $)^{5}$.

ii) Obligatoriedad:

El precedente, de manera general, es obligatorio y tiene efectos generales; sin embargo y eventualmente, la Corte podría modificarlo si verifica situaciones que ameriten el cambio a partir de un caso en concreto, de manera argumentada, garantizando la progresividad y la vigencia del Estado constitucional de derechos y justicia. El pensamiento jurídico de la Corte es de dos clases: De origen y derivado. De origen cuando se construye ex novo, es decir, sin que existan precedentes anteriores; y derivado, cuando toma precedentes anteriores para construirse, modificarse o mantenerse" (Sentencia CCE, 2012).

iii) Utilidad:

La importancia de los criterios jurisprudenciales radica por cuanto ilustra e informa, proporcionándoles antecedentes jurídicos sobre los problemas controvertidos y resueltos aplicables a casos concretos. Actualmente, los precedentes constitucionales tienen trascendental importancia en materia de derechos constitucionales, pues, proporcionan antecedentes jurídicos sobre el tema controvertido [...] Su importancia radica por su contenido, pues son profundamente meditadas, cuanto por su autoridad, puesto que proviene de la más alta Corte de justicia de la República. Por esta doble razón sirve de guía

${ }^{5}$ Constante en ambas sentencias. 
para la recta interpretación y aplicación del derecho en el thema decidendum. Así, las consideraciones jurídicas que anteceden al caso concreto constituyen una condición sine qua non para la buena realización del oficio del juez, pues, en el ejercicio de su potestad jurisdiccional, se fortifica en virtud de los métodos y reglas de la interpretación jurídica que orienta para explicar el problema, para describir lealmente las respuestas, para desentrañar los senderos posibles, para arribar a una solución integral, justa, conveniente, razonable, ponderada y factible (Sentencia CCE, 2015a).

Una vez analizados los factores existentes en el sistema judicial ecuatoriano, la siguiente sección hará una distinción crítica de su valor en el régimen jurídico ecuatoriano.

\section{El valor de la jurisprudencia internacional en el ordenamiento jurídico ecuatoriano}

El problema surge en relación con la Jurisprudencia Internacional, y de ella la del Sistema Interamericano de Protección de Derechos Humanos, materia de este estudio. Al respecto, la Corte Constitucional no plantea ninguna regla particular de precedentes. Su jurisprudencia propone, en el caso de derechos humanos, que los tratados internacionales deben guardar "armonía y conformidad con las normas constitucionales, es decir, sujetarse a esta sin perjuicio de la aplicación de los principios pro ser humano, de no restricción de derechos, de aplicabilidad directa y de cláusula abierta" (Sentencia CCE, 2015b); y propone como fuente creadora de precedentes jurisprudenciales a la Corte Interamericana de Derechos Humanos, entre otros tribunales Internacionales (CCT, 2011).

Pero ¿es la jurisprudencia del SIDH obligatoria Mediante una regla de Derecho dentro del sistema jurídico ecuatoriano si no hay un elemento base para poder ponerla en práctica? En el sistema internacional, hay una serie de reglas que deben tenerse en cuenta como base y que explicamos a continuación.

a) La Jurisprudencia es una fuente auxiliar. El Art. 38 del Estatuto de la Corte Internacional de Justicia propone que las decisiones judiciales y las doctrinas de los publicistas de mayor competencia de las distintas naciones, como medios auxiliares para la determinación para las reglas de Derecho, que sólo pueden ser hechas para los particulares involucrados en el caso (Véase Art. 59).

b) Las normas internacionales son un aporte para el derecho internacional en el sentido de que son "auxiliares, pero no secundarias". Es decir, que ayudan a conocer cuál es el mejor contenido del Derecho y pueden servir para la creación de normas consuetudinarias, sin que ello no las haga superiores a los tratados (Sorensen, 1973, pp. 178-179).

c) La jurisprudencia de los tribunales "es autoritaria y puede ser excluyente o persuasiva en los tribunales nacionales de todos los Estados miembros, pero no es jurídicamente vinculante para ellos" (Shelton, 2009, p. 70).

Entonces, y considerando que el sistema jurídico supranacional es basado en un orden internacional caótico de varias hegemonías en conflicto (Kissinger, 2016), el sistema jurídico busca generar alguna respuesta de forma autorreferencial. $\mathrm{Al}$ respecto, y centrándonos en el sistema interamericano, la respuesta surge a partir de la generación de varias herramientas metodológicas surgidas de la jurisprudencia:

${ }^{6}$ Sentencia de la Corte Constitucional de transición. 
i) Principio de Efecto Útil:

Los Estados partes en la Convención deben garantizar el cumplimiento de las disposiciones convencionales y sus efectos propios (effet utile) en el plano de sus respectivos derechos internos. Este principio se aplica no sólo en relación con las normas sustantivas de los tratados de derechos humanos (es decir, las que contienen disposiciones sobre los derechos (Faundez Ledesma, 1999, p. 60).

ii) Principio de Corpus Iure:

El sistema de protección internacional debe ser entendido como una integralidad, principio recogido en el artículo 29 de la Convención Americana, e impone un marco de protección que siempre da preferencia a la interpretación que más favorezca los derechos de la persona humana, objetivo angular de protección (Sentencia CIDH, 2018, Vélez Loor Vs. Panamá, párrafo 34).

iii) Control de Convencionalidad:

Los jueces y órganos vinculados a la administración de justicia en todos los niveles están en la obligación de ejercer ex officio un "control de convencionalidad" entre las normas internas y la Convención Americana, en el marco de sus respectivas competencias y de las regulaciones procesales correspondientes. En esta tarea, los jueces y órganos vinculados a la administración de justicia deben tener en cuenta no solamente el tratado, sino también la interpretación que del mismo ha hecho la Corte Interamericana, intérprete última de la Convención Americana (Sentencia CIDH, 2012c, Furlan y Familiares Vs. Argentina, párrafo 203).

En nuestro caso, el ordenamiento jurídico ecuatoriano, aceptaría como obligatoria la jurisprudencia del sistema interamericano, considerando que el mismo conjunto jurisprudencial genera obligaciones sobre sí mismas respecto de los Estados que hayan ratificado la Convención Americana de Derechos Humanos. Ese hecho, aunque importante para el presente estudio, no resuelve cómo se elige el precedente judicial para la aplicación de un caso, ni su propósito. Las siguientes secciones buscarán explicar ese elemento a través del concepto de "nichos de conocimiento" en materia judicial.

\section{El caso del SIDH como ejemplo de nicho de conocimiento judicial y categoría de análisis jurídico}

El sistema jurídico sufre una pérdida doctrinaria en el plano del conocimiento relativo a la construcción del sistema de decisión de los jueces. Existe todavía el concepto de que la acción del juez es una "boca muda" de la ley y con ellos se potencia con el colonialismo en los sistemas judiciales nacionales respecto al ordenamiento internacional. Es decir: la idea de que lo generado por un tribunal exterior al país, se considere como más legítimo, por el supuesto de que es un sistema menos viciado que el nacional. El caso de la Corte Interamericana es distinto por cuanto hace un papel de interpretador dinámico (Sentencia CIDH, 2005a, Comunidad Indígena Yakye Axa Vs. Paraguay, párrafo 128) que utiliza todo el sistema de forma permanentemente actualizable, y que ha sido calificado por ella misma como "positivo" por cuanto actualiza las relaciones entre Estados dentro del sistema internacional (Sentencia 
CIDH, 2006b, Masacres de Ituango Vs. Colombia, párrafo 157), y que ese mecanismo se encuentra conforme tanto con la CADH, y la Convención de Viena de Derecho de los Tratados ${ }^{7}$. Pero en ningún momento de su jurisprudencia, se proponen reglas de conducta para utilizar la jurisprudencia del SIDH, más allá de su obligatoriedad.

Para responder cuál es el criterio que el juez del SIDH utiliza en la elección de jurisprudencia hay que tener en cuenta que todo sistema judicial, incluyendo el judicial, es afectado por los procesos de teoría de la administración. En general, los sistemas jurídicos y su manifestación en instancias procesales son una una respuesta administrativa a la profusión de los valores liberales modernos en Occidente (Weber, 2001), basada en un sistema racional y reglado dividido entre los diferentes niveles del Estado (Duso, 2005). Todo este poder es limitado y asegurado por un sistema de normas (Gómez, 1994: 49). El sistema normativo, limita la posibilidad de imponer la voluntad propia a un comportamiento ajeno, y lo modula mediante la "dominación" (Weber, 1954: 323), que diferencia el poder de fuente normativa, del despótico.

En ese orden de cosas, la decisión de un juez se basa en que los procesos cognoscitivos de la persona se canalizan en la forma en que se predicen los sucesos. Dado que el aprendizaje de una persona llega a un límite por una situación determinada, la persona predice los sucesos construyendo réplicas de éstos. En el caso de la jurisprudencia, la persona tendrá una serie de respuestas limitadas, basada en las heurísticas disponibles que se tenga en la realidad y por tal razón realiza "constructos" en su mente, para generar respuestas jurídicas a los casos por el puesto a prueba. En Piscología, el constructo es una representación platónica de conceptos creados ex profeso para explicar la realidad de forma adecuada para cada observador. En tal sentido no existen una idea tal como "prácticas humanas verdaderas", ya que los criterios de verdad o falsedad están contenidos por cada observador.

En tal razón, los constructos son medios para poder entender la realidad a través de "la creación de una estructura con la que adquiere un significado" (Kelly, 1950). Entonces, el juez decide utilizar una serie de estructuras que tienen grados objetivos y probados de funcionamiento en la realidad (respuestas de casos anteriores), basados en estructuras con consenso entre los involucrados en el sistema (normas de los instrumentos internacionales); por lo que, frente a escenarios con racionalidad limitada, se utilizan las normas como un medio para reducir las disonancias cognitivas existentes en una situación determinada. Al contrario, si el juez actúa únicamente bajo escenarios de información incompleta y con una fuerte carga ideológica se puede dejar de lado explicaciones alternativas de la realidad, y generar categorías absolutas que no resisten al análisis científico (Schumpeter, 1969).

El sistema de construcciones de las personas se refiere a procesos dicotómicos, cuyos escenarios son limitados. Frente a esto, el juez escogería la mejor alternativa en sí misma, considerando la mejor extensión y posibilidades de su decisión. El constructo (los casos disponibles para dar legitimidad a sus casos) se vuelve conveniente si se tiene una gama de sucesos limitada, y si su funcionamiento depende de su capacidad de adaptación a nuevas necesidades, por lo que se puede generar un constructo con elementos que aparentemente son incompatibles entre sí, y emplearlo en otras circunstancias en la medida que haya experiencias similares (Kelly, 1950, pp. 50-80). En ese orden de cosas, se puede suponer que cualquier acción del juez tenga un cierto valor, al tratar de generar una herramienta capaz de utilizarse en varios contextos; sin embargo, la experiencia en Latinoamérica supone lo contrario. Sorprende encontrar que entre litigantes, jueces y estudiantes existe una marcada preferencia

\footnotetext{
${ }^{7}$ Cfr. Opinión Consultiva OC-16/99 de 1 de octubre de 1999 "El Derecho a la Información sobre la Asistencia Consular en el Marco de las Garantías del Debido Proceso Legal”. Serie A No. 16, párr. 114, y Atala Riffo y Niñas Vs. Chile, párr. 83.
} 
por apoyar la solidez de los argumentos de la doctrina y no utilizar argumentos jurídicos ya probados en una realidad determinada (López Medina, 2002, p. 156).

Así, un sistema que no obliga al principio del stare decisis (ya que la Corte no tiene ninguna regla que obligue a elegir un precedente jurisprudencial u otro), obliga sí a buscar otra herramienta explicativa, que muestre a) qué casos se escogen para solucionar una determinada temática, y b) por qué se eligen esos casos y se rechazan otros. Esto es importante, ya que, en cualquier organización, una decisión que se basa en pasos interdependientes y acumulativos dan lugar a una elección racional (Bañon, 1997, p. 292).

Dado que no se puede suponer una línea jurisprudencial como tal, lo que debe existir es una acumulación, basada en la prueba y el error, de precedentes que funcionan en varios entornos, los cuales reflejan una serie de reglas generales dentro del sistema y que, en consecuencia, podrían mostrar esos elementos que llamamos jurídicamente corpus iure. En términos de la biología, se denomina construcción de nicho a la alteración del hábitat propio o de otra especie por parte de un organismo vivo (Pocheville, 2015). En los estudios de la cultura se propone como un proceso de adaptación de las construcciones humanas a los entornos. La teoría propone que hay un proceso de "competición excluyente" (551) en el que hay una acción racional de que las construcciones (ideas) realizadas por los individuos es una forma adicional para asegurar su propagación a generaciones futuras (Dawkins, 2016).

Entonces, si tomamos en cuenta esta herramienta, lo que debería suceder es lo siguiente: el juez de la CIDH utilizaría de manera consciente herramientas probadas, que elige de todas las demás disponibles, por una elección racional, ya que prueban un punto de derecho. Entonces, ya que no existe una regla obligatoria de seguimiento del precedente judicial, lo que se hace es que se excluyen todos los demás precedentes que "no resultarían útiles" (según el operador judicial), con lo que se emite una decisión que un segundo operador analizaría en el futuro utilizando un precedente ni obligatorio ni vinculante, sino elegido. Este elemento explicaría varios fenómenos existentes en el SIDH, como la regla de expansión constante del Art. 29 de la Corte Interamericana de Derechos Humanos, ya que no se utilizaría el precedente "correcto", sino el precedente que contenga una interpretación evolutiva, o la carencia de ejercicios de analogías en el SIDH, sino una aplicación directa de la norma.

El siguiente apartado explicará el alcance de la situación en términos cuantitativos; para esto se utilizará el análisis cualitativo-cuantitativo del análisis de redes sociales, que será explicado en la siguiente sección.

\subsection{Análisis de nichos de conocimiento en la jurisprudencia del Sistema interamericano 5.1.1. Metodología}

La metodología del presente proyecto se hará mediante cuatro pasos sucesivos basados en la teoría de Análisis de Redes. El Social Network Analysis o SNA es un mecanismo metodológico que, en suma, sirve para poder explicar el funcionamiento de una construcción humana a partir de los modos en los que se conectan ciertos entes (llamados "nodos"), a través de hechos o actos de conexión llamados "aristas" (Wasserman y Faust, 1994). El análisis de la red de citaciones realizada por los jueces de la Corte Interamericana permitirá explicar si existen nichos de conocimiento: casos que se repiten con regularidad, que soportaron la competencia excluyente de las diferentes formaciones. Para ello, se siguieron las siguientes líneas básicas. 
a) Levantamiento de información de casos. Se eligieron como unidades de análisis los casos de la Corte Interamericana sesionados en el año $2016^{8}$, dado que cumplen dos condiciones: son las decisiones máximas del SIDH; y cuentan con la data —el uso de sentencias anteriores - más avanzada; esto haría que se cuente con unidades de análisis bien delimitadas, como lo exige la doctrina (Rodríguez Treviño, 2013). Toda esta información fue construida en torno a bases de datos de Microsoft Excel, que pueden ser transformadas en archivos CSV (un tipo de documento en formato abierto sencillo para representar datos en forma de tabla) y XLS (diseñado para uso de hojas de cálculo).

b) Levantamiento de red social. La construcción de una red no es un sistema de medición cuantitativa nuevo. La idea de utilizar una manifestación gráfica de interconexiones lo planteaba Simmel y Durkheim (Wasserman y Faust, 1994, p. 36). La gestión de relaciones complejas tuvo sus primeros aportes teóricos en los estudios de Jacob L. Moreno y Helen Jennings (1938). Dado que lo que se quiere demostrar la existencia de regularidad en la jurisprudencia del SIDH, esta herramienta puede ayudar a mostrar las conexiones existentes entre el universo de sentencias consultado (Rodríguez, 1995).

En esta oportunidad se utilizó el software Onodo. Éste es un sistema a través de la web capaz de analizar comportamientos de redes complejas, bajo la hipótesis previa de que los jueces actúan con variables estables de actuación (Tichy, Tushman, y Fombrun, 1979): deben motivar sus sentencias, y por tanto usan normas.

c) Búsqueda de nichos de conocimiento. Si la teoría de redes es cierta, "la experiencia personal de los individuos está estrechamente vinculada con los aspectos de mayor escala de la estructura social, además de por el control de los individuos particulares" (Granovetter, 1973: 1377), es así que los jueces del SIDH deberìan escoger ciertan jurisprudencia con una regularidad que muestre que son elementos "probados" y que resisten su uso en el sistema jurisprudencial.

Este mecanismo tiene una serie de limitaciones: la primera es la misma naturaleza de violaciones de derechos humanos dentro del SIDH. Por ejemplo, el caso de violaciones al debido proceso es posible que se repita, dado que el sistema interamericano es residual, y por lo tanto depende de un agotamiento de recursos internos cumplido no sólo a nivel normativo por el Estado (Sentencia CIDH, 2006d, Ximenes Lopes Vs. Brasil, párrafo 147). Otro elemento a analizar es el universo de estudio, ya que el número de decisiones de la Corte IDH en el 2016 son sólo una parte del corpus iure del sistema interamericano. Sobre esto, surge una única regla del sistema: la aplicación normativa "debe siempre elegirse la alternativa más favorable para la tutela de los derechos protegidos por dicho tratado, según el principio de la norma más favorable al ser humano" (Sentencia CIDH, 2005b, Masacre de Mapiripán Vs. Colombia, párrafo 106).

\subsubsection{Análisis de Redes}

La red resultante de las citaciones en el SIDH, de los casos escogidos es, de acuerdo al Gráfico 1, la siguiente:

\footnotetext{
${ }^{8}$ Andrade Salmón VS. Bolivia; Gómez Vs. Costa Rica; I.V Vs. Bolivia; Miembros De La Aldea Chichupac Y Comunidades Vecinas Del Municipio De Rabinal Vs. Guatemala; Valencia Hinojosa Y Otra Vs. Ecuador; Yarce y otras Vs. Colombia; Canales Huapaya y otros Vs. Perú; Comunidad Campesina De Santa Bárbara Vs. Perú; Galindo Cárdenas y otros Vs. Perú; Quispialaya Vilcapoma Vs. Perú; Duque Vs. Colombia.
} 


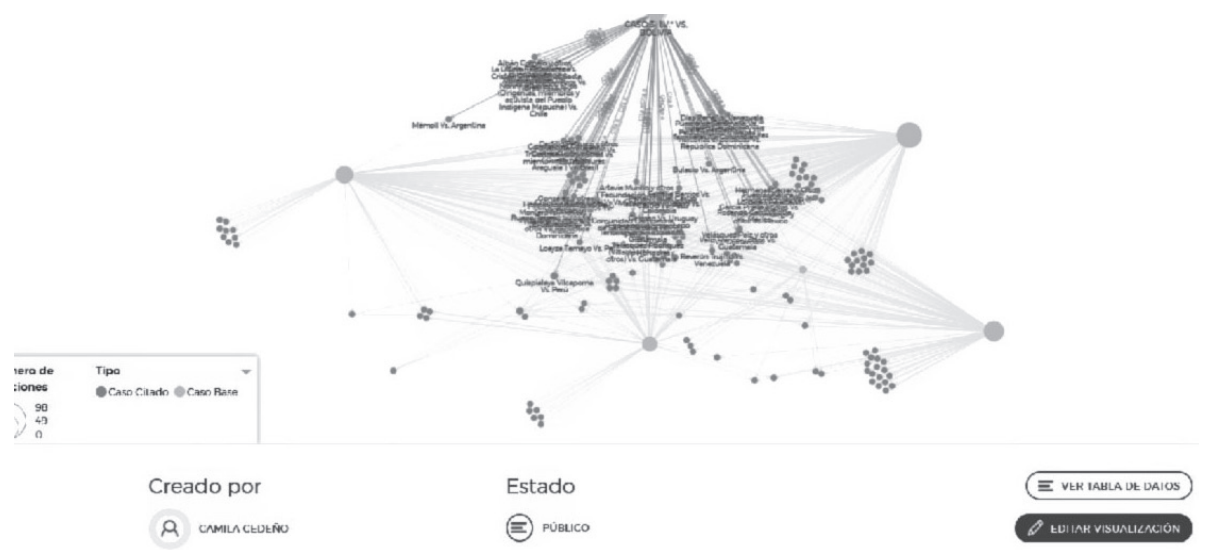

Gráfico 1. Red de Citación Corte Interamericana 2016. Proyección Fructerman-Reingold, mendiante Software Onodo

$\mathrm{Al}$ analizar las 11 sentencias ya referidas de la Corte Interamericana de Derechos Humanos del año 2016, se encontró que la sentencia más citada es el Caso Herrera Espinoza vs. Ecuador con un total de 69 veces que equivale al 5.71\% del total de citas; en segundo lugar está el caso Velásquez Rodríguez vs. Honduras con 66 veces igual al 5.46\%; y en tercer lugar el caso Masacre de Rio Negro vs. Guatemala citado 48 veces con un 3.97\%, sobre un total de 1208 citas entre las 11 sentencias. De la misma jurisprudencia no se encuentran las razones por las cuales estos tres casos son elegidos y no otros. Este fenómeno subraya varias contradicciones del mecanismo interamericano. Por ejemplo, al asumir la jurisprudencia de la CIDH como obligatoria para sí misma (Sentencia CIDH, 2006a, La Cantuta Vs. Perú, párrafo 72) debería cumplir con una acción de codificación normativa: ignora los precedentes en conflicto con la $\mathrm{CADH}$ y la jurisprudencia, y evitar que las precedentes que agudicen en el lenguaje de las leyes escritas. En La Cantuta Vs. Perú, por citar un ejemplo, se propone mostrar que la determinación de las víctimas es "amplia y ajustada a cada caso", lo que puede hacer entender un sistema en que los procedentes son herramientas jurisprudenciales adaptativas, sin mayor elemento de "líneas" o precedentes.

De esta forma, lo que sucede es un fenómeno contrario al del stare decisis: se da una serie de elementos de adaptación normativa, basada en un nicho de conocimiento. Es decir, a diferencia de seguir un encadenamiento de decisiones judiciales a través del tiempo, lo que sucede es que el juez del sistema elige la jurisprudencia sin base normativa sino por otros vectores. Así, tratar los precedentes como superiores a la CADH es basarse en una fuente auxiliar', y generará, como en cualquier sistema de proposiciones lógicas, que la aceptación de una sola contradicción acepte todas las contradicciones, haciendo que cada proposición sea lógicamente indecidible. En tal virtud los precedentes de la Corte IDH no son vinculantes, sino persuasivos, mostrando la línea base a partir de lo que se puede decidir, confirmando a Ferrajoli, quien proponía la necesidad de establecer aquello que puede decidirse o no (2008), en el marco del límite pro homine ${ }^{10}$.

Esto puede desagregarse en los casos analizados. En todos sucede un mismo fenómeno de dos pasos: por un lado, un grupo duro de casos, aparentemente inconexos entre

\footnotetext{
${ }^{9}$ Véase Art. 38 del Estatuto de la Corte Internacional de Justicia.

${ }^{10}$ En este sentido, al interpretar la Convención debe siempre elegirse la alternativa más favorable para la tutela de los derechos protegidos por dicho tratado, según el principio de la norma más favorable al ser humano (Sentencia CIDH, 2005, Masacre de Mapiripán Vs. Colombia, párrafo 106).
} 
sí que son citados como elementos de toma decisión; y por otro, una repetición constante de los casos en la estructura de la sentencia.

a) Andrade Salmón vs. Bolivia con un total de 64 casos citados, en 157 ocasiones diferentes; $8^{11}$ son los casos más citados que representan el 13\%, con un total de 63 citas entre los 8 casos, que equivale al $40 \%$ entre las 157 veces que se citaron.

b) Gómez Murillo vs. Costa Rica con un total de 12 casos citados, en 37 ocasiones diferentes; $3^{12}$ son los casos más citados que representan el $25 \%$, con un total de 28 citas entre los 3 , lo que equivale al $76 \%$ del total de 37 citas.

c) I.V. vs. Bolivia con un total de 61 casos citados, en 199 ocasiones diferentes; 613 son los casos más citados que representan el $10 \%$ entre los 61 casos, con un total de 81 citas entre los 6, lo que equivale al 41\% del total de 199 citas.

d) Miembros de la aldea Chichupac y Comunidades vecinas del municipio de Rabinal vs. Guatemala con un total de 81 casos citados, en 343 ocasiones diferentes; 714 son los casos más citados que representan el $9 \%$ entre los 81 casos, con un total de 139 citas entre los 7 , lo que equivale al $41 \%$ del total de 343 citas.

e) Valencia Hinojosa y otra vs. Ecuador con un total de 47 casos citados, en 117 ocasiones diferentes; 815 son los casos más citados que representan el 17\% entre los 47 casos, con un total de 55 citas entre los 8 , lo que equivale al $47 \%$ del total de 117 citas.

f) Yarce y otras vs. Colombia con un total de 101 casos citados, en 318 ocasiones diferentes; 616 son los casos más citados que representan el 6\% entre los 101 casos, con un total de 94 citas entre los 8 , lo que equivale al $30 \%$ del total de 318 citas.

g) Canales Huapaya y otros vs. Perú con un total de 4 casos citados, en 10 ocasiones diferentes; 217 son los casos más citados que representan el $50 \%$ entre los 4 casos, con un total de 8 citas entre los 2 , lo que equivale al $80 \%$ del total de 10 citas.

h) Comunidad campesina de Santa Bárbara vs. Perú con un total de 2 casos citados, en 4 ocasiones diferentes, equivale al $50 \%$ con 2 citas cada uno.

i) Galindo Cárdenas y otros vs. Perú con un total de 8 casos citados, en 9 ocasiones diferentes; 118 es el caso más citado que representa el 13\% entre los 8 casos, con un total de 2 citas, lo que equivale al $22 \%$ del total de 9 citas.

\footnotetext{
${ }^{11}$ Herrera Espinoza y otros Vs. Ecuador; Quispialaya Vilcapoma Vs. Perú; Tarazona Arrieta y otros Vs. Perú; García Ibarra y otros Vs. Ecuador; Masacre de Santo Domingo Vs. Colombia; Furlan y familiares Vs. Argentina; Granier y otros (Radio Caracas Televisión) Vs. Venezuela; Tenorio Roca y otros Vs. Perú.

${ }^{12}$ Artavia Murillo y otros ("Fecundación In Vitro") Vs. Costa Rica; García Cruz y Sánchez Silvestre Vs. México; Pacheco Teruel y otros Vs. Honduras.

${ }^{13}$ Herrera Espinoza y otros Vs. Ecuador; Velásquez Rodríguez Vs. Honduras; Artavia Murillo y otros ("Fecundación In Vitro") Vs. Costa Rica; Tenorio Roca y otros Vs. Perú; Gonzales Lluy y otros Vs. Ecuador; Velásquez Paiz y otros Vs. Guatemala.

${ }^{14}$ Masacres de Río Negro Vs. Guatemala; Velásquez Rodríguez Vs. Honduras; Comunidad Campesina de Santa Bárbara Vs. Perú; Masacre Plan de Sánchez Vs. Guatemala; Masacre de Las Dos Erres Vs. Guatemala; Tenorio Roca y otros Vs. Perú; García y familiares Vs. Guatemala.

${ }^{15}$ García Ibarra y otros Vs. Ecuador; Herrera Espinoza y otros Vs. Ecuador; Velásquez Rodríguez Vs. Honduras; López Lone y otros Vs. Honduras; Pollo Rivera y otros Vs. Perú; Flor Freire Vs. Ecuador; Castañeda Gutman Vs. México; Montero Aranguren y otros (Retén de Catia) Vs. Venezuela.

${ }^{16}$ Defensor de Derechos Humanos y otros Vs. Guatemala; Herrera Espinoza y otros Vs. Ecuador; Suárez Peralta Vs. Ecuador; Comunidades Afrodescendientes desplazadas de la Cuenca del Río Cacarica (Operación Genésis) Vs. Colombia; Velásquez Paiz y otros Vs. Guatemala; Velásquez Rodríguez Vs. Honduras.

${ }^{17}$ Argüelles y otros Vs. Argentina; Loayza Tamayo Vs. Perú.

${ }^{18}$ Argüelles y otros Vs. Argentina
} 
j) Ovilcapoma vs. Perú con un total de 4 casos citados, en 10 ocasiones diferentes; 219 son los casos más citados que representa el 50\% entre los 4 casos, con un total de 8 citas entre los 2, lo que equivale al $80 \%$ del total de 10 citas.

k) Duque vs. Colombia con un total de 3 casos citados, en 4 ocasiones diferentes; 120 es el caso más citado que representa el $33 \%$ entre los 3 casos, con un total de 2 citas, lo que equivale al $50 \%$ del total de 4 citas.

Del análisis presentado se muestra que: 1) los jueces no tienen una regla jurisprudencial, apuntada más allá de aquellas que por su necesidad estructural (las básicas de la Convención de Viena de Derecho de los Tratados) u obligatorias de aplicación (como la de pro persona) suponen para el funcionamiento del sistema, por lo cual la regla de precedentes no puede demostrar lo que sucede en el caso de la aplicación de la Convención Americana de Derechos Humanos; 2) la jurisprudencia no se explica más que por la temática de los casos que hayan utilizado los casos mayoritarios relacionados con la privación arbitraria de la libertad y torturas así como las violaciones al debido proceso penal y protección judicial; 3) no deja de ser sorprendente que el primer caso del sistema interamericano, Velásquez Rodríguez vs. Honduras, haya tenido tanta importancia, considerando que hay una fuerte jurisprudencia posterior respecto a temas de desaparición forzada y violaciones al derecho a la vida, integridad física y debido proceso. Es posible que el juez que haya aceptado el estándar del caso Velázquez Rodríguez como aceptable haya dejado de aplicar otros casos más actuales, $y$, en consecuencia, se cuente con un nicho de conocimiento respecto al tema, que reemplaza a la línea jurisprudencial o a la interpretación evolutiva.

Este hecho lleva a preguntarnos cuál es el mecanismo de elección de los casos en el SIDH. Es conveniente entender que, de los casos presentados, los casos del SIDH son una autoridad persuasiva más que una de tipo vinculante. Lo que sucede es que, al obligar al juez a que pretenda apartarse de la doctrina probable (existente en nichos de conocimiento claros para el operados judicial) y el precedente judicial a exponer sus razonamientos, se llega a una situación en la que, como expresa la Corte Constitucional Colombiana, "la ratio decidendi de las sentencias [...], en la medida en que se proyecta más allá del caso concreto, tiene fuerza y valor de precedente para todos los jueces en sus decisiones, por lo que puede ser considerada una fuente de derecho que integra la norma" (CCC, 2015), lo que puede ser aunado a la idea de la Corte Constitucional ecuatoriana, que propone que, si un caso tiene como precedente una sentencia en la cual ya se tuteló el derecho reclamado, ha instalado condiciones de predictibilidad, por lo que, el ulterior caso necesariamente debe guardar armonía con el precedente jurisprudencial (CCE, 2016b).

\section{Conclusiones}

El presente estudio ha buscado demostrar, por un lado, las zonas grises existentes en el sistema jurídico internacional respecto a la aplicación de precedentes jurisprudenciales, en un espacio que no tiene una regla de precedente. En segundo lugar, se ha mostrado la tendencia a una aplicación no basada en la regla de interpretación incremental y evolutiva. En tal razón, la tradición legal latinoamericana ha tenido a la jurisprudencia (en la regla general) una excepción como fuente material del Derecho. De hecho, la visión sobre el valor de las decisiones de los jueces ha estado basada en la reputación personal del decisor, o en la importancia del caso, más allá de la existencia de reglas de derecho obligatorias y básicas de la gestión de esa fuente como parte del sistema normativo. En el caso del SIDH, existe una

\footnotetext{
${ }^{19}$ Argüelles y otros Vs. Argentina; Loayza Tamayo Vs. Perú.

${ }^{20}$ Loayza Tamayo Vs. Perú.
} 
cohabitación de dos maneras de aplicar la jurisprudencia: una visión de fuente auxiliar de la creación normativa, y otra que asume sus propios precedentes como obligatorios, sin apuntar en los mismos la idea de una "línea jurisprudencial". Esta segunda se basa en las siguientes condiciones: un grupo de casos que se consideran básicos, pero adaptados en para cada caso; la existencia de condiciones de predictibilidad y la repetición de los mismos dentro de las sentencias sucesivas.

Este texto busca mostrar las bases de un fenómeno y sus alcances jurídicos. Como todo estudio que se basa en la decisión de una autoridad, más aún si consideramos la variedad y riqueza del SIDH, tiene una limitante: la información corresponde a stakeholders (Ackermann y Eden, 2011). En este caso, al no haberse elegido una metodología cualitativa, no se puede responder de manera adecuada a la existencia de las razones personales en los operadores de justicia para elegir un caso o no; ese elemento debería basarse en la posibilidad de aprovechar otras herramientas investigativas que levanten esta información. Ese hecho deberá mostrar, pues, nuevas bases de estudio.

Finalmente, la falta de una regla de precedente jurisprudencial, dentro de un espacio que no la pide, pero que acepta la legitimidad de utilizarla, muestra que la ordenación del sistema internacional depende de lo que los operadores judiciales acepten. En el caso del SIDH, por su importancia y obligatoriedad, se demuestra que el Derecho además de reglas y normas es creencia y necesidad de dar a las palabras una legitimidad que muchas veces no es la prevista en el sistema jurídico, por lo que lo mejor que puede hacerse es, parafraseando a Jonathan Swift en Los viajes de Gulliver, que todo lo que se ha hecho antes puede legalmente hacerse nuevamente. Por lo tanto, debe contarse con especial cuidado de registrar todas las decisiones que antes se hacían contra la justicia común y la razón general de la humanidad, $y$, entre aquellas que logran ser un mecanismo de justicia individual y social, ser un elemento base para mejores decisiones.

\section{Referencias bibliográficas}

Ackermann, F. y Eden, C. (2011). Strategic management of stakeholders: Theory and practice. Long Range Planning, 44 (3), 179-196.

Alexy, R. (1988). Sistemas jurídicos, principios jurídicos y razón práctica. Doxa, 139-151.

Bañon, R. (1997). La nueva administración pública. Madrid: Alianza Editorial.

Casal Hernández, J. (2004). Cosa juzgada y efecto vinculante en la justicia constitucional. Anuario de Derecho Constitucional Latinoamericano, 299-325.

Clemente de Diego, F. (2016). La jurisprudencia como fuente del derecho. Comares: Sevilla.

Dawkins, R. (2016). The Extended Phenotype: The Long Reach of the Gene. London: Oxford University Press.

De Otto, I. (1987). Derecho constitucional. Sistema de fuentes. Barcelona: Ariel.

Duso, G. (2005). El poder: para una historia de la filosofía politica moderna. Editorial Siglo XXI: México D.F.

Faundez Ledesma, H. (1999). El sistema interamericano de protección de los derechos humanos. Aspectos institucionales y procesales. San José: IIDH.

Ferrajoli, L. (2004). Derechos y garantías. La ley del más débil. Madrid: Trotta.

- (2008). La esfera de lo indecidible y la división de poderes. Estudios constitucionales: Revista del Centro de Estudios Constitucionales, 6 (1), 337-343.

García Maynez, E. (1974). Introducción al derecho. México, DF: Porrúa. 
Gómez, E. (1994). Legitimación y racionalización: Weber y Habermas: la dimensión normativa de un orden secularizado. México D.F.: Anthropos Editorial.

Granovetter, M. S. (1973). The Strength of Weak Ties. American Journal of Sociology, 78(6), 1360-1380.

Hernández Walker, L. (2017). Esquizofrenia Judicial: Sentencias Constitucionales vs. Triple Reiteración. Lexis. <https://lexisecuador.wordpress. com/2017/06/01/esquizofrenia-judicial-sentencias-constitucionales-vs-triplereiteracion/\#more-1966>.

Holmes, O. W. (1897). The Path of the Law. Harvard Law Review, 457-478.

Jennings, H. (1938). Statistics of social configurations. Sociometry, 1 (3-4), 342-374..

Kelly, G. A. (1950). The Psychology of Personal Constructs, 2 vols. Nueva York: Norton.

Kennedy, D. (2012). La enseñanza del Derecho como forma de acción política. Buenos Aires: Siglo XXI.

Kissinger, H. (2016). Orden Mundial. Barcelona: Debate.

López Medina, D. (2002). El derecho de los jueces. Bogotá: Legis.

- (2004). Teoría impura del Derecho. La transformación de la cultura jurídica latinoamerica. Bogotá. D.C.: Legis Editores.

Moreno, J. L. (1938). Statistics of social configurations. Sociometry, 1(3-4), 342-374.

Nino, C. S. (2003). Introducción al Análisis del Derecho. Buenos Aires: Astrea.

Pocheville, A. (2015). The Ecological Niche: History and Recent Controversies. En T. Heams,

P. Huneman, G. Lecointre, \& M. Silberstein (eds.). Handbook of Evolutionary Thinking in the Sciences, pp. 547-586. Londres y Nueva York: Springer.

Rodríguez Treviño, J. C. (2013). Cómo utilizar el Análisis de Redes Sociales para temas de historia. Signos históricos, 15 (29), 102-141.

Rodríguez, J. A. (1995). Análisis estructural y de redes. Madrid: Centro de Investigaciones Sociológicas (CIS).

Schumpeter, J. (1969). Ciencia e Ideología. Investigación Económica, 29 (115), 375-377.

Shelton, D. (2009). Soft Law. En D. Armstrong (ed.). Routledge Handbook of International Law (pp. 68-81). Nueva York: Routledge.

Sorensen, M. (1973). Manual de Derecho Internacional Público. México, D.F.: Fondo de Cultura Económica.

Swift, J. (2016). Los viajes de Gulliver. México, D.F.: Fondo de Cultura Económica.

Teubner, G. (1989). Recht als autopoietisches System. Frankfurt am Main: Shrkamp.

Wasserman, S., \& Faust, K. (1994). Social Network Analysis: Methods and Applications. Nueva York: Cambridge University Press.

Weber, M. (1954). Max Weber on Law in Economy and Society. Boston: Harvard University Press.

- (2001). Qué es la burocracia. México D.F.: Ediciones Coyoacán Sociología.

\section{Legislación}

Código Civil Ecuatoriano. (Codificación No. 2005010). Registro Oficial Nº 46 Suplemento, 24 de Junio del 2005.

Constitución de la República del Ecuador. Registro Oficial No 449, 20 de Octubre del 2008. Estatuto de la Corte Internacional de Justicia, Anexo a la Carta de la Organización de las Naciones Unidas, adoptado el 26 de junio de 1945. 


\section{Sentencias}

CCC / Corte Constitucional de la República de Colombia (2015). Sentencia C-621/15, de 30 de septiembre de 2015.

CCE / Corte Constitucional de la República del Ecuador (2016a). № 001-16-SIS-CC, del 6 de Enero de 2016, Magistrado Ponente Principal Ruth Seni Pinoargote. Registro Oficial N 712 Suplemento, 15 de Marzo de 2016.

- (2016b). Nº02-16-SEP-CC, del 6 de Enero de 2016, Magistrado Ponente Víctor Francisco Butiñá Martínez, Registro Oficial No 712 Suplemento, 15 de Marzo de 2016.

- (2015a). N ${ }^{\circ}$ 322-15-SEP-CC, del 30 de Septiembre de 2015, Magistrado Ponente Principal Antonio José Gagliardo Loor, Registro Oficial Nº 654 Suplemento, 22 de Diciembre de 2015.

- (2015b). N 008-15-DTI-CC, del 21 de Octubre de 2015, Magistrado Ponente Principal: DR. Principales MCMS María del Carmen Madonado Sánchez, Registro Oficial Nº 654 Suplemento, 22 de Diciembre de 2015 CCE

- (2012). N 017-12-SIN-CC, del 26 de Abril de 2012, Magistrado Ponente Principal Ruth Seni Pinoargote, Registro Oficial N 743 Suplemento, 11 de Julio de 2012.

CIDH / Corte Interamericana de Derechos Humanos (1987). Velásquez Rodríguez Vs. Honduras. Excepciones Preliminares. Sentencia de 26 de junio de 1987. Serie C No. 1.

- (1997). Loayza Tamayo Vs. Perú. Fondo. Sentencia de 17 de septiembre de 1997. Serie C No. 33.

- (2004). Masacre Plan de Sánchez Vs. Guatemala. Fondo. Sentencia de 29 de abril de 2004. Serie C No. 105.

- (2005a). Comunidad Indígena Yakye Axa Vs. Paraguay. Fondo Reparaciones y Costas. Sentencia 17 de junio de 2005. Serie C No. 125.

- (2005b). Masacre de Mapiripán Vs. Colombia. Sentencia de 15 de septiembre de 2005. Serie C No. 134.

- (2006a) La Cantuta Vs. Perú. Fondo, Reparaciones y Costas. Sentencia de 29 de noviembre de 2006. Serie C No. 162.

- (2006b). Masacres de Ituango Vs. Colombia. Excepción Preliminar, Fondo, Reparaciones y Costas. Sentencia de 1 de julio de 2006. Serie C No. 148.

- (2006c). Montero Aranguren y otros (Retén de Catia) Vs. Venezuela. Excepción Preliminar, Fondo, Reparaciones y Costas. Sentencia de 5 de julio de 2006. Serie C No. 150.

- (2006d). Ximenes Lopes Vs. Brasil. Fondo, Reparaciones y Costas. Sentencia de 4 de julio de 2006. Serie C No. 149.

- (2006d). Castañeda Gutman Vs. México. Excepciones Preliminares, Fondo, Reparaciones y Costas. Sentencia de 6 de agosto de 2008. Serie C No. 184.

- (2009). Masacre de Las Dos Erres Vs. Guatemala. Excepción Preliminar, Fondo, Reparaciones y Costas. Sentencia de 24 de noviembre de 2009. Serie C No. 211.

- (2010). Vélez Loor Vs. Panamá. Excepciones Preliminares, Fondo, Reparaciones y Costas. Sentencia de 23 de noviembre de 2010. Serie C No. 218.

- (2012a). Atala Riffo y Niñas Vs. Chile. Fondo, Reparaciones y Costas. Sentencia de 24 de febrero de 2012. Serie C No. 239.

- (2012b). Artavia Murillo y otros ("Fecundación In Vitro") Vs. Costa Rica. Excepciones Preliminares, Fondo, Reparaciones y Costas Sentencia de 28 noviembre de 2012. Serie C No. 257 
- (2012c). Furlan y Familiares Vs. Argentina. Excepciones Preliminares, Fondo, Reparaciones y Costas. Sentencia de 31 de agosto de 2012. Serie C No. 246.

- (2012d). García y familiares Vs. Guatemala. Fondo Reparaciones y Costas. Sentencia de 29 noviembre de 2012. Serie C No. 258.

- (2012e). Masacre de Santo Domingo Vs. Colombia. Excepciones Preliminares, Fondo y Reparaciones. Sentencia de 30 de noviembre de 2012. Serie C No. 259.

- (2012f). Masacres de Río Negro Vs. Guatemala. Excepción Preliminar, Fondo, Reparaciones y Costas. Sentencia de 4 de septiembre de 2012. Serie C No. 250.

- (2012g). Pacheco Teruel y otros Vs. Honduras. Fondo, Reparaciones y Costas. Sentencia de 27 de abril de 2012. Serie C No. 241.

- (2013). García Cruz y Sánchez Silvestre Vs. México. Fondo, Reparaciones y Costas. Sentencia de 26 de noviembre de 2013. Serie C No. 273.

- (2014) Tarazona Arrieta y otros Vs. Perú Excepción Preliminar, Fondo, Reparaciones y Costas. Sentencia de 15 de octubre de 2014. Serie C No. 286.

- (2015a). Velásquez Paiz y otros Vs. Guatemala. Excepciones Preliminares, Fondo, Reparaciones y Costas. Sentencia de 19 de noviembre de 2015. Serie C No. 307.

- (2015b). Argüelles y otros Vs. Argentina. Interpretación de la Sentencia de Excepción Preliminar, Fondo, Reparaciones y Costas. Sentencia de 23 de junio de 2015. Serie C No. 294.

- (2015c). García Ibarra y otros Vs. Ecuador. Excepciones Preliminares, Fondo, Reparaciones y Costas. Sentencia de 17 de noviembre de 2015. Serie C No. 306.

- (2015d). Gonzales Lluy y otros Vs. Ecuador. Excepciones Preliminares, Fondo, Reparaciones y Costas. Sentencia de 1 de septiembre de 2015. Serie C No. 298.

- (2015e). Granier y otros (Radio Caracas Televisión) Vs. Venezuela. Excepciones Preliminares, Fondo, Reparaciones y Costas. Sentencia de 22 de junio de 2015. Serie C No. 293.

- (2016a). I.V. vs. Bolivia. Excepciones Preliminares, Fondo, Reparaciones y Costas. Sentencia de 30 de noviembre de 2016. Serie C No. 329.

- (2016b) Quispialaya Vilcapoma Vs. Perú. Interpretación de la Sentencia de Excepciones Preliminares, Fondo, Reparaciones y Costas. Sentencia de 21 de noviembre de 2016. Serie C No. 320.

- (2016c). Andrade Salmón vs. Bolivia. Fondo, Reparaciones y Costas. Sentencia de 1 de diciembre de 2016. Serie C No. 330.

- (2016d). Canales Huapaya y otros vs. Perú. Interpretación de la Sentencia de Excepciones Preliminares, Fondo, Reparaciones y Costas. Sentencia de 21 de noviembre de 2016. Serie C No. 321.

- (2016e). Caso Herrera Espinoza vs. Ecuador. Excepciones Preliminares, Fondo, Reparaciones y Costas. Sentencia de 1 de septiembre de 2016. Serie C No. 316.

- (2016f). Comunidad campesina de Santa Bárbara vs. Perú. Interpretación de la Sentencia de Excepciones Preliminares, Fondo, Reparaciones y Costas. Sentencia de 21 de noviembre de 2016. Serie C No. 324.

- (2016g). Duque vs. Colombia. Interpretación de la Sentencia de Excepciones Preliminares, Fondo, Reparaciones y Costas. Sentencia de 21 de noviembre de 2016. Serie C No. 322.

- (2016h). Flor Freire Vs. Ecuador. Excepción Preliminar, Fondo, Reparaciones y Costas. Sentencia de 31 de agosto de 2016. Serie C No. 315.

- (2016i). Galindo Cárdenas y otros vs. Perú. Interpretación de la Sentencia de Excep- 
ciones Preliminares, Fondo, Reparaciones y Costas. Sentencia de 21 de noviembre de 2016. Serie C No. 323.

- (2016j). Gómez Murillo vs. Costa Rica. Sentencia de 29 de noviembre de 2016. Serie C No. 326.

- (2016k). Herrera Espinoza y otros Vs. Ecuador. Excepciones Preliminares, Fondo, Reparaciones y Costas. Sentencia de 1 de septiembre de 2016. Serie C No. 316.

- (20161). López Lone y otros Vs. Honduras. Interpretación de la Sentencia de Excepción Preliminar, Fondo, Reparaciones y Costas. Sentencia de 2 de septiembre de 2016. Serie C No. 317.

- (2016m). Miembros de la aldea Chichupac y Comunidades vecinas del municipio de Rabinal vs. Guatemala. Excepciones Preliminares, Fondo, Reparaciones y Costas. Sentencia de 30 de noviembre de 2016. Serie C No. 328.

- (2016n). Pollo Rivera y otros Vs. Perú. Fondo, Reparaciones y Costas. Sentencia de 21 de octubre de 2016. Serie C No. 319.

- (2016o). Tenorio Roca y otros Vs. Perú. Excepciones Preliminares, Fondo, Reparaciones y Costas. Sentencia de 22 de junio de 2016. Serie C No. 314.

- (2016p). Valencia Hinojosa y otra vs. Ecuador. Excepciones Preliminares, Fondo, Reparaciones y Costas. Sentencia de 29 de noviembre de 2016. Serie C No. 327

- (2016q). Yarce y otras vs. Colombia. Excepción Preliminar, Fondo, Reparaciones y Costas. Sentencia de 22 de noviembre de 2016. Serie C No. 325.

- (2016r). Quispialaya Vilcapoma vs. Perú. Interpretación de la Sentencia de Excepciones Preliminares, Fondo, Reparaciones y Costas. Sentencia de 21 de noviembre de 2016. Serie C No. 320.

\section{Resoluciones y opiniones consultivas}

CCT / Corte Constitucional de Transición de la República del Ecuador (2011). Resolución Administrativa No. 004-10-AD-CC, de 5 de agosto de 2011.

CIDH / Corte Interamericana de Derechos Humanos (1999). El Derecho a la Información sobre la Asistencia Consular en el Marco de las Garantías del Debido Proceso Legal. Opinión Consultiva OC-16/99 de 1 de octubre de 1999. Serie A No. 16. 